\title{
Message from the Guest Editors of the Special Issue on Entry, Descent, and Landing of Tianwen-1—China's First Mission to Mars
}

\author{
Xiangyu Huang ${ }^{1}$, Yu Jiang ${ }^{2}$, and Xiangyuan Zeng ${ }^{3}$ \\ 1. Beijing Institute of Control Engineering, Beijing 100094, China \\ 2. Xi'an Satellite Control Center, Xi'an 710043, China \\ 3. Beijing Institute of Technology, Beijing 100081, China \\ (C) The Author(s) 2022
}

Dear authors and readers,

Mars is the outer neighboring planet to our planet, the Earth, which has long been the destination of the deep space exploration. Through decades of exploration since the 1960s, mankind has learned a lot about Mars. Two main scientific reasons for exploring Mars at least can be summarized, including the search for life, and understanding the planet's evolution. Particularly, Mars can help us to discover the evolution of the Earth and its history, in other words, learning more about our home planet. Of course, going to Mars is very challenging, while orbiting and landing on Mars are even harder. By considering all Martian missions, more than 50 percent of landing attempts have failed where the thin Martian atmosphere makes descent tricky. As such, dynamics and control of the Martian orbiter and the lander is significant to fulfill the successful separation and landing.

Tianwen-1 is the China's first mission by accomplishing Martian orbiting/landing/roving in a single flight proposed by China National Space Administration. The spacecraft was launched on July 23, 2020 and entered Martian orbit on February 10, 2021. On May 15, 2021, the successful soft landing of the lander/Zhurong rover made China the second nation to realize the Martian surface touchdown and roving. The scientific objectives of Tianwen- 1 mission are: (1) mapping the morphology and geological structure of Mars, (2) investigating surface soil characteristics and water-ice distribution, (3) analyzing the surface material composition, (4) measuring the ionosphere as well as the characteristics of the Martian climate and environment at the surface, and (5) perceiving the physical fields and internal structure of Mars.

This special issue collects seven papers, ranging from entry, descent, and landing GNC system design to the descent trajectory reconstruction, from aerodynamic design to the Martian atmosphere reconstruction, etc. All authors are from the Tianwen-1 mission team. The latest results and key techniques to guarantee the up-to-date success of the mission are reported by focusing on the astrodynamics part of the mission.

All contributors to this special issue are highly appreciated with fresh outcomes and new achievements of the mission.

Sincerely,

Xiangyu Huang

Yu Jiang

Xiangyuan Zeng

Open Access This article is licensed under a Creative Commons Attribution 4.0 International License, which permits use, sharing, adaptation, distribution and reproduction in any medium or format, as long as you give appropriate credit to the original author(s) and the source, provide a link to the Creative Commons licence, and indicate if changes were made.

The images or other third party material in this article are included in the article's Creative Commons licence, unless indicated otherwise in a credit line to the material. If material is not included in the article's Creative Commons licence and your intended use is not permitted by statutory regulation or exceeds the permitted use, you will need to obtain permission directly from the copyright holder.

To view a copy of this licence, visit http://creativecommons.org/licenses/by/4.0/.

$\bowtie$ X. Huang, huangxyhit@sina.com; Y. Jiang, jiangyu_xian_china@163.com;

X. Zeng, zeng@bit.edu.cn 